\title{
An Empirical Study on Influencing Factors of Online Behavior of Patients Accepting the Health Care System
}

\author{
Yuan HUANG ${ }^{1, a}$, Ying $\mathrm{CHEN}^{2, \mathrm{~b}}$, Xin-Xin YU ${ }^{1, \mathrm{c}}$, Yan $\mathrm{LI}^{1, \mathrm{~b}}$, Meng-Meng CHEN ${ }^{1, \mathrm{e}}$, \\ Yang-Yang $\mathrm{HU}^{1, \mathrm{f}}$, Xiao-Long $\mathrm{MA}^{1, \mathrm{~g},{ }^{*}}$ and Zhang-Guo SHEN ${ }^{1, h}$ \\ ${ }^{1}$ School of Business, Huzhou University, Huzhou, Zhejiang, China \\ ${ }^{2}$ School of Information Engineering, Huzhou University, Huzhou, Zhejiang, China \\ a2573692760@qq.com, ${ }^{\mathrm{b}} 193799311 @ q q . c o m,{ }^{c} 1023106595 @ q q . c o m,{ }^{d} 1142996244 @ q q . c o m$, \\ e2038981015@qq.com, ${ }^{1} 2038981015 @ q q . c o m, 91 x m 2007 @$ sohu.com, hszgxx@hutc.zj.cn
}

${ }^{*}$ Corresponding author

Keywords: Mobile medical treatment, TAM, SPSS, SEM.

\begin{abstract}
With the improvement of people's living standard nowadays, people's awareness of their own health and the demand for health care knowledge are also increasing. Basing on an in-depth analysis of the existing issues in the development of online medical system, this paper constructs the six-factor TAM model. A questionnaire survey was carried out in Hangzhou. Zhejiang Province, and 414 valid questionnaires were collected. The reliability and the validity of the questionnaire were analyzed with the help of SPSS statistical analysis software. The structured equation model (AMOS) was used to empirically test the conceptual model. Research results showed that the medical convenience, convenient communication, patient's privacy and digital management system using conveniently for patients have a remarkable effort on promoting patients to accept online medical care. Based on the research, the countermeasures and suggestions of the development of online medical system were putting forward.
\end{abstract}

\section{Introduction}

With the progress of Internet technology, Internet technology has changed the way people seek medical and health care information. Internet technology has become an important way for Internet users to learn about medical and health care knowledge. The business of the early Internet medical information service website has emerged, the medical information service website international first appeared, such as " Health door ", "Health, Netscape" there are many time-honored brands in China, such as " Medicine for seeking medical treatment net", "39 Health Network".

With the deep integration of the Internet and traditional industries, the online medical information service platform is increasing and rapidly grabbing the medical market. Although these online medical information service platforms have somewhat alleviated the problem of "difficult and expensive to see a doctor" in our country the survey shows that only about 27 percent of the netizens feels that online medical information service websites can respond to their problems with a timely manner, while the dissatisfaction rate is up to $41 \%$, while $35 \%$ of the Internet users consider that the reliability of the response is low. Therefore, from the point of view of patient behavior, based on the TAM model, combined with the actual situation of China's online medical system, from the perspective of patient perception, this paper constructs a model that affects the factors of patients' use of online medical system. In order to improve the construction of online medical information system and provide reference from the development and progress of online medical information system, the problems of online medical information system can be found by testing and analysis. 


\section{Literature Review}

\section{The Concept of Online Medical Treatment}

Online medical treatment refers to the use of medical and health App based on mobile terminal systems such as Android and Apple to provide medical services and information through mobile communications technology.With the rapid development of online medical treatment, the traditional way of going to the hospital has been gradually changed. People can use the APP of medical health information to consult their condition and adopt the doctor's advice at any time as well as provide access to a variety of health knowledge related to their own health.Online medical treatment provides a useful way for people to solve the medical treatment under the condition of the "difficult and expensive to see a doctor" in our country. It will save a lot of money and time in order to line up, register and even go on the hospital by all means of vehicles. What's more, it can effectively guide people to develop good hygiene habits.

\section{Problems in On-line Medical Information System}

As the platform for the communication between patients and doctors in the Internet era, online medicine occupies a very important position. Many hospitals now have built online medical information systems. However, there are many problems in the process of patients' use, such as imperfect functionality, incomplete data, a poor user experience, etc.

In view of the above problems, many scholars have made research on the development and function of design of online medical system.For example, Qiao Liang (2010) and other scholars have proposed the design and implementation of remote online Sketchpad based on the hybrid technology of XML and AJAX ;Shen Tingting (2013) and other scholars introduced the relevant functions of online medical information system according to the research and analysis of Hangzhou; Shao Shuang (2014) and other scholars have compared the service mode, operation mode and profit model of some online medical websites in China, and then put forward some suggestions of online medical development path. But all these studies have overlooked that the development of online medical system should take the patient at the center, instead of just improving the system itself. This kind of online medical system lacks, lacking attention to the patient's need, must not be accepted by the patient very well.

\section{Model Structure}

\section{Hypothesis based on Technology Acceptance Model}

In 1989, Davis proposed the Technology Adoption Model, which is intended to interpret and predict whether the users will be able to accept information systems after they use them. The model holds that the two most important factors affecting the acceptance of the system are perceived usefulness (the degree to which the user considers the use of the system useful to him) and ease of use (perceived by the user as the degree of difficulty in using the system). At present, there are many empirical studies show that the TAM model is reliable. Therefore, this paper will apply to TAM model to study online medical information system, and explains the factors that predict the impact on patients' use of online medical information system from the perspective of patient perception.

\section{Model Structure}

Based on literature review, summarized the research target, this article selects medical convenience, the system USES simple, privacy protection, medical experience sharing, rich disease information and disease digital management as the latent variables. This paper proposes the following hypotheses:

H1a: The convenience of medical treatment has a positive effect on perceived usefulness of online medical treatment.

$\mathrm{H} 1 \mathrm{~b}$ : The convenience of medical treatment has a positive impact on perceived online medical ease of use. 
H2a: The use of the system has a positive effect on perceived usefulness of online medical treatment.

$\mathrm{H} 2 \mathrm{~b}$ : The use of the system has a positive effect on the perceived online medical ease of use.

H3a: Patient privacy protection has a positive effect on perceived usefulness of online medical treatment.

H3b: Patients' privacy protection has a positive impact on perceived online medical ease of use.

H4a: Sharing medical experience has a positive impact on the perceived usefulness of online medical care.

$\mathrm{H} 4 \mathrm{~b}$ : Sharing medical experience has a positive impact on perceived online medical ease of use.

H5a: The adequate information about the disease has positive influence on the usefulness of online medical treatment.

$\mathrm{H} 5 \mathrm{~b}$ : The adequate information about the disease has a positive impact on the perceived ease of use of online medical treatment.

H6a: The digital management of the disease has a positive influence on the perceived usefulness of online medical treatment.

H6b: The digital management of the disease has a positive impact on the perception of online medical ease of use.

H7: Perceived online medical ease of use has a positive impact on the perceived usefulness of online medical care.

H8: Perception of the usefulness of online medical care has a positive impact on the use of system decisions.

H9: Perceived online medical ease of use has a positive impact on the use of system decisions.

The research model constructed in this paper is shown in figure 1:

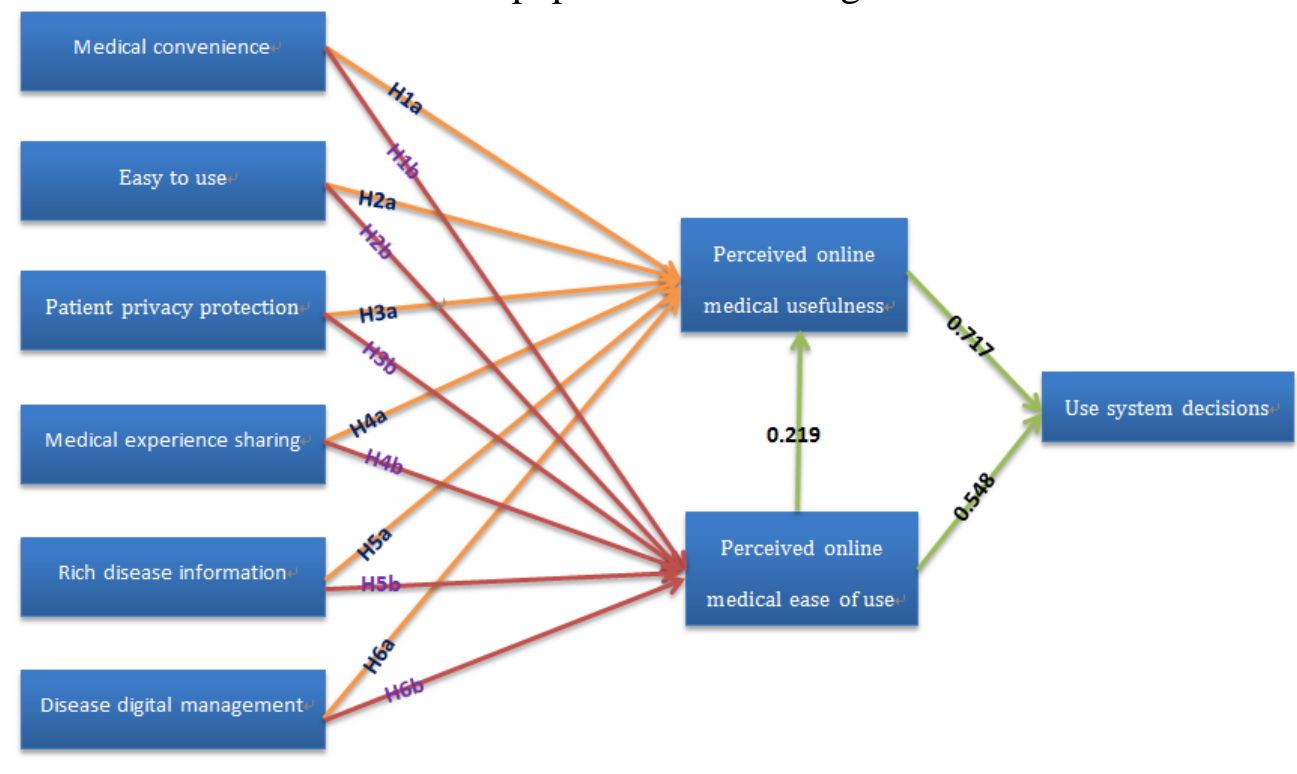

Fig. 1 Research model

\section{Assuming that Explanation}

H1a: Medical convenience refers to the extent to which patients can benefit from using the online medical system. Patients use the online medical system when they think it is useful for their own convenience. Let's say I have a positive impact.

H1b: Patients use the online medical system when they think it is easy to use. Let's say I have a positive impact.

$\mathrm{H} 2 \mathrm{a}$ : The ease of use of the system refers to the extent to which patients use the online medical system to determine whether the system is easy to learn. When patients think that using an online medical system is easy and easy to learn, patients will use it when they are useful. Let's say I have a positive impact. 
$\mathrm{H} 2 \mathrm{~b}$ : When patients think it is easy to learn how to use an online medical system, they will use it when they are easy to use. Let's say I have a positive impact.

$\mathrm{H} 3 \mathrm{a}$ : Patient privacy protection refers to the private data generated when patients use the online medical system. Patients use the online medical system when they think it is useful to protect their privacy. Let's say I have a positive impact.

$\mathrm{H} 3 \mathrm{~b}$ : Patients use the online health care system to protect their privacy when patients think it is easy to use. Let's say I have a positive impact.

H4a: Sharing medical experience to refer to patients can use online medical system to share medical experience with other users. Patients use the online medical system when they think it is useful to share a medical experience. Let's say I have a positive impact.

H4b: Patients use an online medical system when they think it's easy to share with their experience. Let's say I have a positive impact.

H5a: The information about the disease is rich in the information that patients get when they use the online medical system. Patients use it when they think that the rich illness information on the online medical system is useful to them. Let's say I have a positive impact.

H5b: Patients use it when they think that the rich information on the online health care system is easy of them to use. Let's say I have a positive impact.

H6a: The digital management of the disease is the digital management of the patient's disease data in the online medical system. When the patient thinks that the online medical system's disease data management is useful for him, it will be used. Let's say I have a positive impact.

H6b: It is used when patients think that the online medical system's disease data management is easy to use. Let's say I have a positive impact.

H7: When patients perceive the ease with which an online medical system is used, the online medical system is considered useful. Let's say I have a positive impact.

H8: When the patient perceives the online medical system as useful, it will allow the patient to accept the online medical system. Let's say I have a positive impact.

H9: When patients perceive the ease with which an online medical system is used, patients are given access to an online medical system. Let's say I have a positive impact.

\section{Study Design}

\section{Research Objects and Locations}

The subjects in this study were determined to be patients, and the investigation was based on hospitals. We investigated the factors affecting patients' acceptance of online medical system in the first Hospital of Zhejiang Province, the first people's Hospital of Hangzhou, the Zhongshan Hospital of Zhejiang Province, the second Hospital of Zhejiang Medical Service and the people's Hospital of Zhejiang Province.

\section{Design of Questionnaire}

The questionnaire in this paper was designed based on literature and research model, the validity of all the items in the questionnaire was tested, and all the results were obtained in the previous literature. In this paper, the questionnaire includes two parts. The first part is the basic information questionnaire, which mainly obtains the patient's basic information such as gender, age, duration of illness, occupation, monthly income, and hospitalization. The second part of the questionnaire consist of 6 impact indicators: convenience for medical treatment, convenient use of online medical system, no leakage of privacy by online medical system, sharing of discussion experience with patients, adequate information of illness related to oneself, and good digital management of illness. The evaluation criteria are measured according to the Likert five-point scale, that is, very agreeable, agree, uncertain, disagree, very disagree. After the design of the questionnaire was completed, a small - scale re - study was conducted, and then the questionnaire was modified appropriately to form the final questionnaire. A total of 439 questionnaires were collected, and 25 invalid questionnaires were excluded, and 414 valid questionnaires were obtained. 


\section{Data Processing}

\section{Reliability Analysis}

Reliability refers to the characteristics of a test score or the result of the measurement, not the best or the measuring tool itself.The commonly used method of reliable tests in the Likert scale is "Cornbach's alpha" coefficient.In this study, the 0.7 value proposed by Pedersen (1994) was used as the test standard.

Table 1 Cronbach'Alpha value table

\begin{tabular}{ccc}
\hline Cronbach's $\alpha$ & $\begin{array}{c}\text { Based on standardized terms } \\
\text { Cronbach's } \alpha\end{array}$ & The number of item \\
.816 & .803 & 19 \\
\hline
\end{tabular}

Table 2 Cronbach'Alpha detailed list

\begin{tabular}{lcc}
\hline \multicolumn{1}{c}{ Influence factors } & $\begin{array}{c}\text { The number of measurable } \\
\text { variables }\end{array}$ & $\begin{array}{c}\text { The reliability } \\
\text { of } \alpha\end{array}$ \\
\hline Medical convenience & 2 & 0.879 \\
Easy to use & 2 & 0.776 \\
Patient privacy protection & 2 & 0.796 \\
Medical experience sharing & 3 & 0.654 \\
Rich disease information & 2 & 0.793 \\
Disease digital management & 2 & 0.704
\end{tabular}

According to the criteria above, the 0.7 value can be used as the test standard. From table 1 , we can see that the overall reliability coefficient of the test questionnaire is 0.803 , indicating that the whole questionnaire have good internal consistency and reliability; It can be seen from table 2 that the reliability coefficient, the alpha, value of each part of the test questionnaire are all greater than 0.6 , indicating that the stability and affirmation of each part are good, so the designed questionnaire is reliable.

\section{Validity Analysis}

Validity is divided into constructive validity and content validity.This research used SPSS21.0 software to analyze the construction validity.First, we used Bartlett sphere tests and KMO measure to determine whether the data were suitable for factor analysis. Then we got the factor structure matrix of each item based on factor analysis, and compared it to the test value.According to the study of KAISER, the value of KMO above 0.9 is very good, more than 0.8 are good, more than 0.7 are moderate, more than 0.6 are poor, and less than 0.5 cannot be analyzed by factor.

Table 3 Detailed variable KMO and Bartlett's inspection

\begin{tabular}{lcccc}
\hline Variates & $\begin{array}{c}\text { KMO samples } \\
\text { detection }\end{array}$ & $\begin{array}{c}\text { J Approximate chi } \\
\text { square value }\end{array}$ & $\begin{array}{c}\text { Bartlett test } \\
\text { Df(Degrees of } \\
\text { freedom) }\end{array}$ & $\begin{array}{c}\text { Sig } \\
\text { (significance) }\end{array}$ \\
\hline Medical convenience & 0.500 & 82.160 & 1 & .000 \\
\hline Easy to use & 0.500 & 42.199 & 1 & .000 \\
\hline Patient privacy protection & 0.500 & 49.489 & 1 & .000 \\
\hline Medical experience sharing & 0.636 & 39.966 & 1 & .000 \\
\hline Rich disease information & 0.500 & 41.199 & 1 & .000
\end{tabular}

The results of the validity test are all in conformity with the standard of validity test, and the validity of the structure validity of the questionnaire is qualified. In a word, the questionnaire has reached its standard and has a good overall validity. 


\section{Model Validation}

In this paper, the influencing factors of patients' acceptance behavior in online medical system are difficult to measure directly and difficult to avoid subjective measurement errors.Karl G. Joreshog, a Swedish statistician who put forward the structural equation models SEMMO-SEM as an analytical tool that can provide an observable and processing tool and incorporate errors into the model. So SEM was used to analyze the main factors of patients' acceptance of online medical system in this study. The relationship between exogenous variables and potentially endogenous variables was investigated by using confirmatory hypothesis. In this structural equation, it was allowed to have a certain measurement error on the variables and the potential variables have multiple measurements,so that the fitting degree of the model and data could be evaluated by many indices in the structural equation.Then used the SEM to test the research model and AMOS21.0 software was used to process the data.The results of the study are shown in figure 2 below:

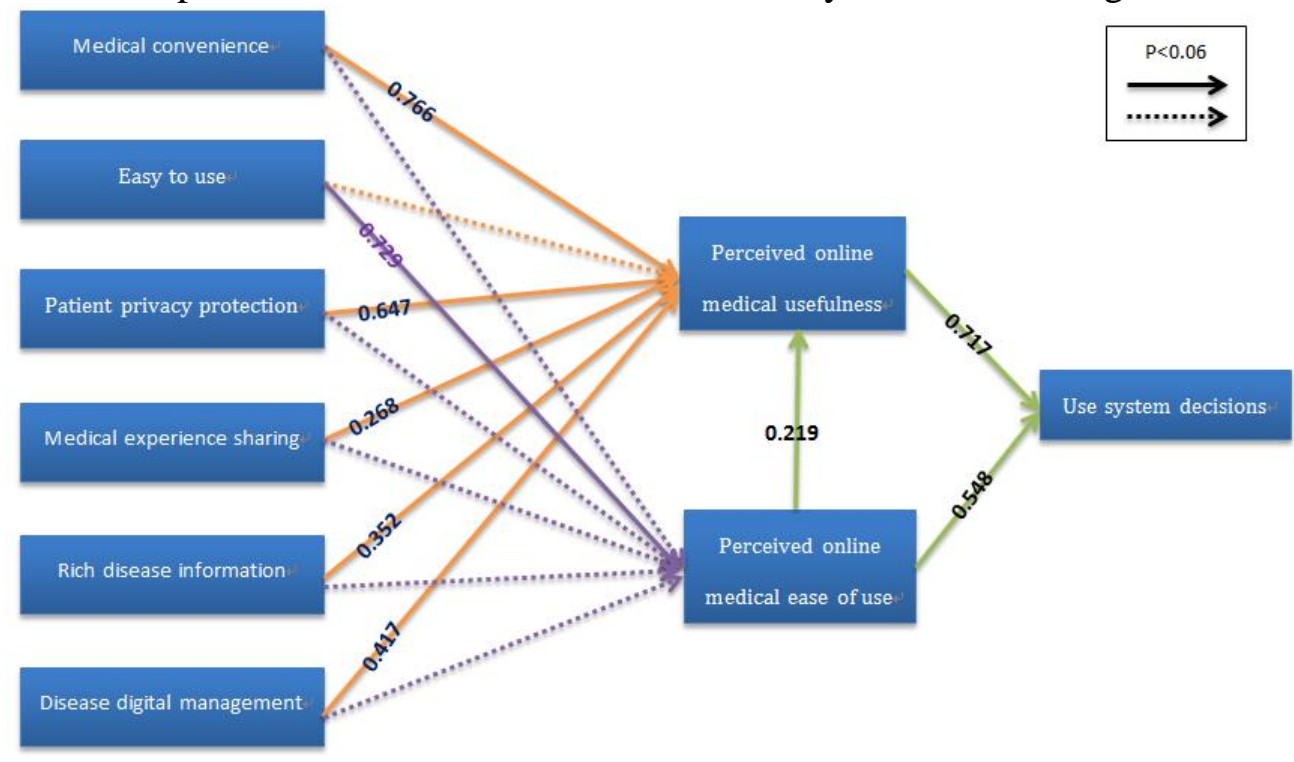

Fig.2 Model test results

From the analysis of the above experimental results, it can be seen from figure 2 that when the significant level is 0.05 ,these relationships are significant which are the relationship between the convenience of medical treatment, the protection against patients' privacy, the sharing of medical experience, adequate information on the state of illness, the digital management of the disease and the usefulness of the patients' perception of online medical treatment; And the relationship between the convenience of using and the usability of the patients' perception of online medical treatment as well as the relationship between the usefulness and the usability of the patients' perception of online medical treatment. What's more, the usefulness of the patient's perception of online medical treatment is significantly related to their use of system decisions. Meanwhile, the usability of the patient's perception of online medical treatment is also significantly related to their use of system decisions. So the hypothesis of $1 \mathrm{a}, 2 \mathrm{~b}, 3 \mathrm{a}, 4 \mathrm{a}, 5 \mathrm{a}, 6 \mathrm{a}, 7,8,9$ was all be confirmed.

However, there is no significant relationship between the convenience of medical treatment, the protection against patients' privacy, the sharing of medical experience, adequate information on the state of illness, the digital management of the disease and the usefulness of the patients' perception of online medical treatment; and so is the relationship between the convenience of using and the usability of the patients' perception of online medical treatment . Therefore,the hypothesis of $1 \mathrm{~b}, 2 \mathrm{a}, 3 \mathrm{~b}, 4 \mathrm{~b}, 5 \mathrm{~b}, 6 \mathrm{~b}$ was not to be supported.

\section{Conclusion}

The sample scope of this questionnaire is the first Hospital of Zhejiang Province, the first people's Hospital of Hangzhou,Zhongshan Hospital of Zhejiang Province, the second Hospital of 
Zhejiang Medicine and the people's Hospital of Zhejiang Province. And the SEM was used to study the main factors that affected the patients ' acceptance of online medical system based on 414 questionnaires. The results show that these relationships are significant which are the relationship between the convenience of medical treatment, the protection against patient's privacy, the sharing of medical experience, adequate information on the state of illness, the digital management of the disease and the usefulness of the patient's perception of online medical treatment; And the relationship between the convenience of using and the usability of the patients' perception of online medical treatment as well as the relationship between the usefulness and the usability of the patients' perception of online medical treatment. What's more, the usefulness of the patients' perception of online medical treatment is significantly related to their use of system decisions. Meanwhile, the usability of the patient's perception of online medical treatment is also significantly related to their use of system decisions. However, There is no significant relationship between the convenience of medical treatment, the protection against patients' privacy, the sharing of medical experience, adequate information on the state of illness, the digital management of the disease and the usefulness of the patients' perception of online medical treatment; and so is the relationship between the convenience of using and the usability of the patients' perception of online medical treatment.

\section{Management and Enlightenment}

Through the above research, this paper thinks that online medical treatment should pay more attention to the construction of the following aspects to make patients easily to accept online medical information system:

(1)The time of the online reservation and real-time station number is accurate. The green channel is being opened to provide emergency services for emergency patients, so that the information about the patients can be acquired early and hospital can make preparations in advance to provide more timely and effective first aid treatments.

(2)Increase the way of treatment and balance the medical resources. Make adjustments on condition of the crowds of people in the hospital while extremely poor situation in the clinic. At the same time, the way of assessment should be used to absorb doctors from second-line hospitals so that makes full use of doctors that have ability but be put on ice.

(3)Construct the platform of medical knowledge popularization and patient communication.To facilitate the real - time acquisition of professional medical knowledge for patients and build a bridge between patients to exchange information.

\section{Acknowledgement}

This work is supported by the National College Students' innovation and entrepreneurship training programs(project number:201710347025,project name: Based on the reading curriculum standards and conformance testing track evaluation system), the Zhejiang Province College students science and technology innovation activities plan project(project number:2017R427030,project name: Excellent family Sharing Community) and This work was funded by the Zhejiang Natural Science Foundation, project NO. Y18G030049.

\section{References}

[1] Hao Min, Wang Pu, Tang Jinhui. The enlightenment of German hospital management system to Chinese hospital management [J]. PLA hospital management journal, 2011,18 (8): 798-800.

[2] Zhang Yanqing. The interpretation of "healer's benevolence" [J]. Chinese medical ethics, 2012,25 (2): 257-258.

[3] Shao Jiaqing, Gan Zhenhua, Sun Wei, etc. Analysis of technological innovation on improving the driving force of medical service quality [J]. Management reform review, 2014,35 (6A): 67-69. 
[4] Qiao Liang, Chen Xin, Song Wenqiang. Development of remote medical online drawing board based on B/S mode [J]. Medical and health equipment, 2010, 31(9):54-56.

[5] Shen Tingting, Zhang Guoxin, Cao Zhuoxiang, etc. The significance of an online medical service platform and construction mode analysis -- based on the investigation and analysis of Hangzhou [J]. Modern property • Modern economy, 2013, 12(9):83-85. 